\title{
Droplet breakup in the square microchannel with a short square constriction to generate slug flow
}

\author{
Xiaoda Wang ${ }^{1}$, Yuanyuan $\mathrm{Liu}^{2}$, Dayu $\mathrm{Liu}^{3}$, Xuehui $\mathrm{Ge}^{2}$, Ling $\mathrm{Li}^{2}$, and Ting $\mathrm{Qiu}^{2}$ \\ ${ }^{1}$ Fuzhou University \\ ${ }^{2}$ Affiliation not available \\ ${ }^{3}$ Institute of Technology of University of Paris-Saclay
}

January 8, 2022

\begin{abstract}
Droplet breakup in micro-constrictions is an important phenomenon in industrial applications. This work aimed to investigate the droplet breakup in the square microchannel with a short square constriction to generate the slug flow, which drew little attention before. Mechanism analysis indicated that this breakup process included the shear-force-dominated, squeezing-forcedominated, and pinch-off stages. Non-uniform daughter droplets were generated in the constriction with their interface restricted in the horizontal and perpendicular directions by the microchannel walls. The average relative deviation of the daughter droplet size was $<30 \%$, much lower than that for the breakup with the daughter droplet restricted only in one direction. An empirical equation with a deviation of $<20 \%$ was provided to show the dependence of the daughter droplet size on the operation conditions. The comparison results suggested that the different restriction effects of microchannel wall on daughter droplets led to the different breakup mechanisms in different constrictions.
\end{abstract}

\section{Hosted file}

manuscript.docx available at https://authorea.com/users/454567/articles/552153-dropletbreakup-in-the-square-microchannel-with-a-short-square-constriction-to-generate-slug-

flow 